3. Kulkarni S, Barbagli G, Kirpekar D, Mirri F, Lazzeri M: Lichen sclerosus of the male genitalia and urethra: surgical options and results in a multicenter international experience with 215 patients. Eur Urol. 2008; Jul 30. [Epub ahead of print]

4. Patterson JM, Chapple CR. Surgical techniques in substitution urethroplasty using buccal mucosa for the treatment of anterior urethral strictures. Eur Urol. 2008; 53: 1162-71.

\author{
Dr. Karl-Dietrich Sievert \& \\ Dr. Arnulf Stenzl \\ Department of Urology \\ Eberhard-Karls-University Tuebingen \\ Tuebingen, Germany \\ E-mail: arnulf.stenzl@med.uni-tuebingen.de
}

\title{
UROLOGICAL ONCOLOGY
}

Toxicities associated with the administration of sorafenib, sunitinib, and temsirolimus and their management in patients with metastatic renal cell carcinoma

Bhojani N, Jeldres C, Patard JJ, Perrotte P, Suardi N, Hutterer G, Patenaude F, Oudard S, Karakiewicz PI

Cancer Prognostics and Health Outcomes Unit, University of Montreal, Montreal, QC, Canada

Eur Urol. 2008; 53: 917-30

Objective: To provide a systematic review of the side effects associated with sorafenib, sunitinib, and temsirolimus and to provide an outline of possible preventive or therapeutic measures.

Methods: We performed a PubMed-based systematic review of side effects associated with the three agents and relied on product monographs and prescribing information to provide an outline of treatments aimed at reducing these toxicities.

Results: Side effects range from $<1 \%$ to $72 \%$. Grade $3 / 4$ side effects are less common and range from $<1 \%$ to $13 \%$ for sorafenib, $<1 \%$ to $16 \%$ for sunitinib, and $1 \%$ to $20 \%$ for temsirolimus. Overall, sunitinib causes the most grade $3 / 4$ side effects and sorafenib causes the fewest grade 3/4 side effects, although head-to-head trials are required to compare safety profiles of all three kinase inhibitors. Virtually all side effects can be managed effectively.

Conclusion: Prevention, recognition, and prompt management of side effects are of key importance and avoid unnecessary dose reductions, which may undermine treatment efficacy.

\section{Editorial Comment}

Three targeted medical therapies against metastatic renal cancer have recently been approved and are more and more widely used. Either as a therapist himself or in counseling his/her patients in further medical therapy the urologist faces a new generation of drugs with unfamiliar side-effects. The article focuses on the side effects of sunitib, sorafenib and temsirolimus as reported in the literature from all phase I, II and III studies. This article is worthwhile reading, profound and detailed and is recommended for every urologist dealing with this tumor entity.

The authors not only describe the side-effects of treatment but also give detailed advice on the management of hematologic, systemic or endocrinologic, cardiac, gastrointestinal, cutaneous and laboratory adverse events. Moreover, dose-modifications are suggested. Finally, a helpful questionnaire to monitor the patents course and standardized prescriptions are given. 
Again, strongly advised reading for everyone dealing with medical therapy against renal cancer.

\author{
Dr. Andreas Bohle \\ Professor of Urology \\ HELIOS Agnes Karll Hospital \\ Bad Schwartau, Germany \\ E-mail:boehle@urologie-bad-schwartau.de
}

\title{
Prepubic urethrectomy during radical cystoprostatectomy \\ Joniau S, Shabana W, Verlinde B, Van Poppel H \\ Department of Urology, University Hospitals Leuven, Leuven, Belgium \\ Eur Urol. 2007; 51: 915-21
}

Objectives: In muscle-invasive bladder cancer, the risk of developing a urethral recurrence after cystectomy varies between $4 \%$ and $18 \%$, especially when an ileal conduit is performed. For this reason, some authors advocate a urethrectomy in these indications. At our center, we developed the technique of prepubic urethrectomy. We assessed the feasibility and implications of this technique over $20 \mathrm{yr}$ of use.

Patients and Methods: We retrospectively analyzed the medical files of 180 consecutive male patients who underwent a urethrectomy simultaneously with cystectomy for invasive bladder cancer between 1985 and 2005. We describe our technique step-by-step, and present possible technical difficulties and complications of urethrectomy.

Results: The mean increase in operative time with the prepubic urethrectomy over cystoprostatectomy alone was 17 min (range: 15-25). Postoperative complications occurred in $10(5.5 \%)$ patients. A subcutaneous penile haematoma was noted in four $(2.2 \%)$ patients, two of whom needed a circumcision later on. A scrotal haematoma was seen in five $(2.7 \%)$ patients; two needed a surgical drainage and three were treated conservatively. A prepubic collection was noted in one patient who was also treated conservatively. No thromboembolic or neurologic complications were encountered. Conclusions: When a urethrectomy is indicated, it can best be performed by using the prepubic approach, because it is easier and less time-consuming than the perineal approach, and has very limited and easily manageable complications.

\section{Editorial Comment}

Urologic surgeons familiar with radical cystectomy are confronted with the risk of urethral recurrences. Indications for this procedure vary between different authors, but in cases with multifocal carcinoma in situ or clear infiltration of the prostate simultaneous urethrectomy mostly is recommended.

These authors describe an elegant and time-saving procedure to perform prepubic urethrectomy and report their 20 years of experience.

Remarkably, in 180 cases of en-bloc urethrectomy together with radical cystectomy the authors experienced only 10 patients with complications.

From my own experience in many such cases I can only recommend this operative procedure and would advice anyone with experience in operative urology to thorough reading of this article.

Dr. Andreas Bohle

Professor of Urology

HELIOS Agnes Karll Hospital

Bad Schwartau, Germany

E-mail: boehle@urologie-bad-schwartau.de 